\title{
A critical analysis of the tools to evaluate neuropsychiatric lupus
}

Kristina E N Clark ${ }^{1}$

Camilla N Clark ${ }^{2}$

Anisur Rahman ${ }^{1}$

${ }^{1}$ Centre for Rheumatology, Division of Medicine, University College London, London, UK

2St George's Hospital, London UK

Corresponding Author: anisur.rahman@ucl.ac.uk

Address for Correspondence and reprint requests:

Professor A Rahman

Centre for Rheumatology Research

UCL Division of Medicine, Rayne Building $4^{\text {th }}$ Floor, 5 University Street

London

Tel: 00442031082168

Fax: 00442031082152

There are no conflicts of interest to be declared.

Anisur Rahman is supported by the National Institute for Health Research University

College London Hospitals Biomedical Research Centre. 


\section{Abstract}

Neuropsychiatric symptoms occur commonly in patients with systemic lupus erythematosus (SLE), but they are not always due to active disease. It is crucial to identify cases that are due to active SLE so that appropriate treatment can be instituted. There is no single serological or imaging test that distinguishes active neuropsychiatric SLE (NPSLE) from NP manifestations caused by other factors such as infection. Most patients with NPSLE have generalised features of disease activity. Raised anti-dsDNA and low C3 complement levels are often seen, but are not an invariable guide. The presence of antiphospholipid antibodies is more suggestive of thrombotic than inflammatory causation. A number of other autoantibody tests have been proposed as biomarkers for NPSLE, but results in clinical studies have been inconsistent and none has so far entered routine clinical practice. Cerebrospinal fluid features and magnetic resonance imaging (MRI) appearances are non-specific in NPSLE, but are useful in excluding other causes of NP symptoms. Newer MRI sequences show promise for distinguishing new NPSLE activity from previous damage and recent research suggests these may correlate with changes in cognitive function in patients with SLE. However, formal cognitive testing is seldom carried out in the acute setting.

\section{Key words}

Systemic lupus erythematosus, neuropsychiatric, autoantibodies, magnetic resonance imaging

\section{Running title}

Evaluation of NPSLE 


\section{Introduction}

Neuropsychiatric (NP) events in SLE can affect between $21-95 \%$ of patients with

SLE (1). This wide range of prevalence figures across different studies arises from the fact that manifestations recognised as NPSLE are very varied. The American College of Rheumatology (ACR) has defined 19 NPSLE syndromes (2). NPSLE can vary from symptoms such as mild mood disorder to psychosis or status epilepticus. NP involvement is rarely a presenting feature of SLE (3), but is associated with lower patient-reported health related quality of life indices $(1,4)$. NPSLE accounts for up to $19 \%$ of deaths in patients with SLE (5). Thus it is very important to be able to identify which NP symptoms in a patient with SLE are due to active disease and require treatment with corticosteroids/immunosuppression.

\section{Current tools for analysis of NPSLE}

\section{Clinical Assessment}

Between $40-50 \%$ of NP events occur in the presence of generalised active SLE (3). It is rare for NPSLE to occur in the absence of any other form of disease activity. Global disease activity measured by SLEDAI-2K was found to be significantly higher in patients with NPSLE than patients with SLE (6), mainly in patients with central nervous system (CNS) NPSLE or diffuse NP syndromes. 
Serum analysis

The typical picture of elevated erythrocyte sedimentation rate (ESR) with normal CRP levels characteristic of active SLE may also be seen in NPSLE (7), and the additional presence of raised anti-double stranded DNA (anti-dsDNA) and reduced complement (8) is a strong pointer to active disease. However, a normal ESR or negative serology does not exclude CNS lupus and raised inflammatory markers can occur in other conditions causing NP symptoms in patients with SLE, such as CNS infection.

Antibodies play an important role in disease pathogenesis in SLE (9). It has been postulated that NPSLE may be a result of dysregulation of the blood brain barrier (10). Currently, the only autoantibody tests in routine clinical use for evaluating NPSLE are anti-dsDNA and antiphospholipid antibodies.

High titres of double stranded DNA antibodies are seen in $70 \%$ of patients with active NPSLE symptoms (7), and support a diagnosis of globally active SLE, but they are not specific for NPSLE syndromes.

Antiphospholipid antibodies (aPL) measured in clinical practice encompass anticardiolipin (aCL), lupus anticoagulant (LAC) and anti-beta2glycoprotein I (anti$\left.\beta_{2} \mathrm{GPI}\right)$. Their association with both focal and diffuse presentations of NPSLE is well 
studied, including stroke, seizures, epilepsy, migraine headaches as well as cognitive impairment $(11,12)$. aCL has also been found to be associated with cognitive impairment (13), but this association is not upheld in all studies (14). However, overall $\mathrm{aCL}$ is more strongly associated with $\operatorname{NPSLE}(11,15,16)$ when compared with LAC, whereas LAC has the stronger association with cerebrovascular disease, especially stroke or sinus thrombosis (17). aPL are also associated with increased risk of transverse myelopathy, chorea, seizure and migrainous headache (18).

There is a relationship between aPL antibodies and cognitive impairment. In one study cognitive deficits were three times more common in APS and SLE with aPL than healthy controls $(80 \%, 75 \%$ and $16 \%$ respectively) (19).

It is important, however, to note that positive tests for aPL are typically not helpful in decisions about immunosuppression. They may instead inform decisions regarding whether to anticoagulate the patient.

CSF Analysis

$30-90 \%$ of patients with active NPSLE are found to have mild non-specific abnormalities in their CSF, typically modestly raised WCC (usually a lymphocytosis) and elevated protein with normal glucose (7). In a study of 41 patients (7), abnormal CSF results were associated with a poorer outcome, with higher morbidity and mortality. 
Aseptic meningitis is rare, accounting for up to $4.3 \%$ of NPSLE presentations (20). CSF often demonstrates normal or only mildly low glucose and a lower lymphocyte count than in viral meningitis. Abnormal CSF is indicative of a worse outcome. Oligoclonal bands are found in $15-85 \%$ of patients with SLE, in the absence of other supporting evidence for multiple sclerosis.

Overall, lumbar puncture is not absolutely necessary in the management of possible NPSLE, especially with clear signs of SLE activity (raised anti-dsDNA and low C3). However, CSF analysis may be invaluable where the differential diagnosis between NPSLE and infection is unclear.

Cerebral Imaging

Computer-assisted Tomography (CT). CT is not a reliable tool in NPSLE, its main use is to enable differentiation from infarcts and haemorrhages during an acute presentation of stroke.

Magnetic Resonance Imaging. MRI remains the imaging modality of choice. It is a very sensitive tool for picking up inflammatory or ischaemic changes secondary to SLE, and to exclude alternative diagnoses. There is no robust evidence supporting particular abnormalities on MRI that are specific for NPSLE (21). Abnormal scans tend to be associated with focal, and normal scans with more diffuse, neurological presentations (7). The most common cerebral MRI abnormalities described in patients with SLE are white-matter hyperintensities (8-75\% of patients) in a 
predominantly fronto-parietal distribution $(22,23)$, and cerebral atrophy $(9-67 \%)(24$, 25). Focal lesions or non-specific changes seen on MRI (including vascular infarcts) do not reliably correlate with disease severity (26). MRI techniques able to detect tissue microstructural changes include diffusion tensor imaging (DTI) and magnetization transfer imaging (MTI) and appear to show some correlation with the severity of NPSLE (26).

In a retrospective study, $40 \%$ of NPSLE patients had completely normal cerebral MRI scans $(21,24)$. The most frequent abnormality identified in the remaining $60 \%$ was microangiopathy, predominantly in the frontal and parieto-occipital regions, which in turn correlated with cognitive dysfunction, low CH50 and LAC. 13.7\% of NPSLE had evidence of microbleeds and $11.1 \%$ lacunar infarcts, again associated with LAC.

Large vessel disease is estimated to affect about $13 \%$ of NPSLE presentations (21) with $50 \%$ of these patients having more than one large-vessel infarct. Primary cerebral vasculitis (both focal or widespread) on $\mathrm{MRI}$ remains rare, was estimated to affect $6.5 \%$ of SLE patients having MRIs (21), and was associated with markers of global disease activity, most specifically low complement.

\section{Cognitive testing}

Since cognitive testing is perceived to be time consuming and often not immediately available, it is seldom requested in suspected acute NPSLE. However, studies have demonstrated cognitive dysfunction in $20-80 \%$ of patients with SLE across a broad 
range of cognitive domains including; attention, executive function and speed of processing, learning and memory, visuospatial skills and psychomotor function (27). The most commonly reported changes were in learning and attention with studies attributing some of these changes to alterations in subcortical white matter and fronto-parietal function $(27,28)$.

One of the main challenges of cognitive testing in these patients is the interaction with increasing age, major depression and steroid use (29). Cognitive testing must be interpreted in the context of these and other factors which are likely to affect performance.

\section{Potential new serum assays for analysis of NPSLE}

Anti-ribosomal $P$ antibodies (anti- $P$ )

A positive anti-P test (30) shows high specificity for SLE, occurs in $15-20 \%$ of patients with SLE and high titres may be a marker of active disease(31). Elevated anti-P was reported to have a strong association, both in serum and CSF, with lupus related psychosis $(17,30,32,33)$. Other studies claimed that high anti-P levels were very specific for depression and psychosis, especially when present with anti- $\beta_{2} \mathrm{GPI}$ (34). Detection of anti-P in the serum can predate the first episode of psychosis by a median of 5.69 years (17). However, a large international meta-analysis (35) with 1537 patients did not support the use of anti-P either as a marker of NPSLE as a whole or for psychosis or mood disorders. 
Anti-N-methyl-D-aspartate receptor subtype 2 (anti-NR2) antibodies

Anti-dsDNA antibodies cross-react with NR2 receptors in the CNS, suggesting a potential role in NPSLE (36). Originally high anti-NR2 was thought to be strongly associated with depression, short term memory and learning impairment (37-39), but these findings failed to be reproduced despite multiple attempts (29).

Although titres of anti-NR2 were initially thought to be associated with significantly lower performance scores in sustained attention and executive function in SLE, they are not consistently found to be a reliable biomarker for cognitive dysfunction $(29,40)$ and this association did not hold up in a large meta-analysis (34).

Anti-NR2 antibodies are found in the CSF of a third of SLE patients (41), which is higher than other autoimmune diseases. During acute CNS presentations or septic meningitis, CSF anti-NR2 titres increase more than serum levels (42). However, antiNR2 are rarely detectable if anti-dsDNA is undetectable.

Other antibodies

Anti-GABAR $R_{B 1 b}$ and anti-GABAR $R_{B 2}$ antibodies were found exclusively in SLE (but only in $15 \%$ of patients) in one small study (43). There was also a significant association between the titres and SLEDAI scores. 
Anti-endothelial antibodies are found in the serum of $17-75 \%$ of patients with SLE (44), and are associated with psychiatric disorders in NPSLE, most specifically psychosis and depression.

Anti-neuronal antibodies. A recent large meta-analysis (34) of 41 trials found significant increases in the serum titres of aCL, LAC, aPL, anti-P antibodies and antineuronal antibodies in patients with NP-SLE when compared to patients with SLE, but not NPSLE. In CSF, only anti-neuronal antibody titres were significantly increased in patients with NPSLE.

Nitrated Nucleosome levels (NN)

Both nucleosome levels and overall level of serum protein nitration are elevated in patients with active SLE. Thus Croca et al (45) developed a capture ELISA to measure NN levels. Serum NN was significantly higher in patients with SLE than in healthy people or in patients with other autoimmune diseases. Serum NN titres were twice as high in patients with NPSLE flares than in those without NPSLE, but this was a small study in only 49 patients and the data have yet to be confirmed.

\section{Potential new imaging techniques for analysis of NPSLE}

Newer MRI sequences such as diffusion weighted images (DWI) and apparent diffusion coefficient (ADC) may be helpful in diagnosis and prognosis. When 
assessing for posterior reversible encephalopathy syndrome, vasogenic oedema (which carries a better prognosis) is identified with hypointensity or isointensity on DWI and hyperintensity on ADC. Conversely, hyperintensity on DWI and hypointensity on ADC are associated with irreversible outcomes (46).

Novel MRI techniques able to detect tissue microstructural changes, including diffusion tensor imaging (DTI) and magnetization transfer imaging/ magnetisation transfer spectroscopy, appear to show some correlation with changes seen in NPSLE (26).

Magnetic resonance spectroscopy (MRS) measures cell-specific metabolites, and has been correlated with cognitive dysfunction in NPSLE (47). Recently published data combines diffusion weighted imaging techniques with MRS (DW-MRS) to allow examination of cell and microstructural changes (26). Relatively specific glial changes were shown as a marker of previous NPSLE, but patients with active NPSLE were not included. This technique may be incorporated with other markers of microstructural damage (DTI, MTI and Susceptibility weighted imaging) to aid in the diagnostic process.

Functional MRI (fMRI)

fMRI has demonstrated that patients with SLE may maintain cognitive performance through compensatory recruitment of regions where function is preserved. The commonest finding is increased signal in regions involved in working memory and executive function, mainly fusiform gyrus, prefrontal cortex, parietal regions, 
supplementary motor area and caudate body $(48,49)$, when compared to healthy controls.

It may be possible to use fMRI to distinguish between acute presentations of NPSLE, and more chronic disease, by increased resting state functional connectivity in the fronto-parietal cortex in the former, and decreased functional connectivity in the latter [58]. However, most studies have only included small numbers of SLE patients. No correlation has been reported between increased signal on $\mathrm{fMRI}$ and disease activity measured through serum biomarkers $(28,50)$. The most reproducible finding in SLE is lack of effective integration of functional brain regions during tasks.

There is altered cerebral blood flow (CBF) on fMRI in NPSLE, with abnormal CBF being seen in diffuse and focal NPSLE in a regional distribution. This has also been shown to normalise with resolution of symptoms (51).

PET/SPECT

PET-CT and MR spectroscopy were found to have $100 \%$ sensitivity in active NPSLE, but specificity is low compared to MRI (52).

PET and SPECT scans show non-specific changes in NPSLE. However they can detect subclinical CNS involvement and reveal irregularities in patients with cerebral involvement, buts normal MRI scans (53). 


\section{Conclusion}

There are no specific individual serological, CSF or neuroimaging findings that enable a definite diagnosis of NPSLE to be made. However, by integrating the results of clinical and serological analysis together with imaging one can ascertain the likelihood that the patient's clinical presentation is related to active NPSLE rather than other causes such as infection. Cognitive testing and neurophysiology are not often required in the acute phase of NPSLE, but may be more useful in detecting long-term sequelae.

\section{References}

1. Hanly JG, Urowitz MB, Jackson D, Bae SC, Gordon C, Wallace DJ, et al. SF-36 summary and subscale scores are reliable outcomes of neuropsychiatric events in systemic lupus erythematosus. Annals of the rheumatic diseases. 2011;70(6):961-7.

2. Hanly JG. ACR classification criteria for systemic lupus erythematosus: limitations and revisions to neuropsychiatric variables. Lupus. 2004;13(11):861-4.

3. Bertsias GK, loannidis JP, Aringer M, Bollen E, Bombardieri S, Bruce IN, et al. EULAR recommendations for the management of systemic lupus erythematosus with neuropsychiatric manifestations: report of a task force of the EULAR standing committee for clinical affairs. Annals of the rheumatic diseases. 2010;69(12):2074-82.

4. Mak A, Cheung MW, Chiew HJ, Liu Y, Ho RC. Global trend of survival and damage of systemic lupus erythematosus: meta-analysis and meta-regression of observational studies from the 1950s to 2000s. Seminars in arthritis and rheumatism. 2012;41(6):830-9.

5. Zirkzee EJ, Huizinga TW, Bollen EL, van Buchem MA, Middelkoop HA, van der Wee NJ, et al. Mortality in neuropsychiatric systemic lupus erythematosus (NPSLE). Lupus. 2014;23(1):31-8.

6. Morrison E, Carpentier S, Shaw E, Doucette S, Hanly JG. Neuropsychiatric systemic lupus erythematosus: association with global disease activity. Lupus. 2014;23(4):370-7.

7. Joseph FG, Lammie GA, Scolding NJ. CNS lupus: a study of 41 patients. Neurology. 2007;69(7):644-54.

8. Magro-Checa C, Schaarenburg RA, Beaart HJ, Huizinga TW, Steup-Beekman GM, Trouw LA. Complement levels and anti-C1q autoantibodies in patients with neuropsychiatric systemic lupus erythematosus. Lupus. 2016;25(8):878-88.

9. Greenwood DL, Gitlits VM, Alderuccio F, Sentry JW, Toh BH. Autoantibodies in neuropsychiatric lupus. Autoimmunity. 2002;35(2):79-86.

10. Hanly JG. Diagnosis and management of neuropsychiatric SLE. Nature reviews Rheumatology. 2014;10(6):338-47. 
11. Afeltra A, Garzia P, Mitterhofer AP, Vadacca M, Galluzzo S, Del Porto F, et al. Neuropsychiatric lupus syndromes: relationship with antiphospholipid antibodies. Neurology. 2003;61(1):108-10.

12. Mok CC, To CH, Mak A. Neuropsychiatric damage in Southern Chinese patients with systemic lupus erythematosus. Medicine. 2006;85(4):221-8.

13. Hanly JG, Hong C, Smith S, Fisk JD. A prospective analysis of cognitive function and anticardiolipin antibodies in systemic lupus erythematosus. Arthritis and rheumatism. 1999;42(4):728-34.

14. Efthimiou P, Blanco M. Pathogenesis of neuropsychiatric systemic lupus erythematosus and potential biomarkers. Modern rheumatology. 2009;19(5):457-68.

15. Mikdashi J, Handwerger B. Predictors of neuropsychiatric damage in systemic lupus erythematosus: data from the Maryland lupus cohort. Rheumatology (Oxford, England). 2004;43(12):1555-60.

16. Borowoy AM, Pope JE, Silverman E, Fortin PR, Pineau C, Smith CD, et al. Neuropsychiatric lupus: the prevalence and autoantibody associations depend on the definition: results from the 1000 faces of lupus cohort. Seminars in arthritis and rheumatism. 2012;42(2):179-85.

17. Hanly JG, Urowitz MB, Su L, Bae SC, Gordon C, Clarke A, et al. Autoantibodies as biomarkers for the prediction of neuropsychiatric events in systemic lupus erythematosus. Annals of the rheumatic diseases. 2011;70(10):1726-32.

18. Wong RC, Favaloro EJ. Clinical features, diagnosis, and management of the antiphospholipid syndrome. Seminars in thrombosis and hemostasis. 2008;34(3):295-304.

19. Coin MA, Vilar-Lopez R, Peralta-Ramirez I, Hidalgo-Ruzzante N, Callejas-Rubio JL, OrtegoCenteno $\mathrm{N}$, et al. The role of antiphospholipid autoantibodies in the cognitive deficits of patients with systemic lupus erythematosus. Lupus. 2015;24(8):875-9.

20. Kampylafka El, Alexopoulos H, Kosmidis ML, Panagiotakos DB, Vlachoyiannopoulos PG, Dalakas MC, et al. Incidence and prevalence of major central nervous system involvement in systemic lupus erythematosus: a 3-year prospective study of 370 patients. PLoS One.

2013;8(2):e55843.

21. Sarbu N, Alobeidi F, Toledano P, Espinosa G, Giles I, Rahman A, et al. Brain abnormalities in newly diagnosed neuropsychiatric lupus: systematic MRI approach and correlation with clinical and laboratory data in a large multicenter cohort. Autoimmunity reviews. 2015;14(2):153-9.

22. Gal Y, Twig G, Mozes O, Greenberg G, Hoffmann C, Shoenfeld Y. Central nervous system involvement in systemic lupus erythematosus: an imaging challenge. The Israel Medical Association journal : IMAJ. 2013;15(7):382-6.

23. Luyendijk J, Steens SC, Ouwendijk WJ, Steup-Beekman GM, Bollen EL, van der Grond J, et al. Neuropsychiatric systemic lupus erythematosus: lessons learned from magnetic resonance imaging. Arthritis and rheumatism. 2011;63(3):722-32.

24. Sibbitt WL, Jr., Brooks WM, Kornfeld M, Hart BL, Bankhurst AD, Roldan CA. Magnetic resonance imaging and brain histopathology in neuropsychiatric systemic lupus erythematosus. Seminars in arthritis and rheumatism. 2010;40(1):32-52.

25. Zivadinov R, Shucard JL, Hussein S, Durfee J, Cox JL, Bergsland N, et al. Multimodal imaging in systemic lupus erythematosus patients with diffuse neuropsychiatric involvement. Lupus. 2013;22(7):675-83.

26. Ercan E, Magro-Checa C, Valabregue R, Branzoli F, Wood ET, Steup-Beekman GM, et al. Glial and axonal changes in systemic lupus erythematosus measured with diffusion of intracellular metabolites. Brain : a journal of neurology. 2016;139(Pt 5):1447-57.

27. Tay SH, Mak A. Anti-NR2A/B Antibodies and Other Major Molecular Mechanisms in the Pathogenesis of Cognitive Dysfunction in Systemic Lupus Erythematosus. International journal of molecular sciences. 2015;16(5):10281-300. 
28. Hou J, Lin Y, Zhang W, Song L, Wu W, Wang J, et al. Abnormalities of frontal-parietal restingstate functional connectivity are related to disease activity in patients with systemic lupus erythematosus. PLoS One. 2013;8(9):e74530.

29. Harrison MJ, Ravdin LD, Lockshin MD. Relationship between serum NR2a antibodies and cognitive dysfunction in systemic lupus erythematosus. Arthritis and rheumatism. 2006;54(8):251522.

30. Briani C, Lucchetta M, Ghirardello A, Toffanin E, Zampieri S, Ruggero S, et al. Neurolupus is associated with anti-ribosomal $P$ protein antibodies: an inception cohort study. Journal of autoimmunity. 2009;32(2):79-84.

31. Zandman-Goddard G, Chapman J, Shoenfeld Y. Autoantibodies involved in neuropsychiatric SLE and antiphospholipid syndrome. Seminars in arthritis and rheumatism. 2007;36(5):297-315.

32. Bertsias GK, Boumpas DT. Pathogenesis, diagnosis and management of neuropsychiatric SLE manifestations. Nature reviews Rheumatology. 2010;6(6):358-67.

33. Hirohata S, Arinuma Y, Takayama M, Yoshio T. Association of cerebrospinal fluid antiribosomal $p$ protein antibodies with diffuse psychiatric/neuropsychological syndromes in systemic lupus erythematosus. Arthritis research \& therapy. 2007;9(3):R44.

34. Ho RC, Thiaghu C, Ong H, Lu Y, Ho CS, Tam WW, et al. A meta-analysis of serum and cerebrospinal fluid autoantibodies in neuropsychiatric systemic lupus erythematosus. Autoimmunity reviews. 2016;15(2):124-38.

35. Karassa FB, Afeltra A, Ambrozic A, Chang DM, De Keyser F, Doria A, et al. Accuracy of antiribosomal $P$ protein antibody testing for the diagnosis of neuropsychiatric systemic lupus erythematosus: an international meta-analysis. Arthritis and rheumatism. 2006;54(1):312-24.

36. DeGiorgio LA, Konstantinov KN, Lee SC, Hardin JA, Volpe BT, Diamond B. A subset of lupus anti-DNA antibodies cross-reacts with the NR2 glutamate receptor in systemic lupus erythematosus. Nature medicine. 2001;7(11):1189-93.

37. Omdal R, Brokstad K, Waterloo K, Koldingsnes W, Jonsson R, Mellgren SI. Neuropsychiatric disturbances in SLE are associated with antibodies against NMDA receptors. European journal of neurology. 2005;12(5):392-8.

38. Diamond B. Antibodies and the Brain: Lessons from Lupus. Journal of immunology (Baltimore, Md : 1950). 2010;185(5):2637-40.

39. Lauvsnes MB, Omdal R. Systemic lupus erythematosus, the brain, and anti-NR2 antibodies. Journal of neurology. 2012;259(4):622-9.

40. Massardo L, Bravo-Zehnder M, Calderon J, Flores P, Padilla O, Aguirre JM, et al. Anti-Nmethyl-D-aspartate receptor and anti-ribosomal-P autoantibodies contribute to cognitive dysfunction in systemic lupus erythematosus. Lupus. 2015;24(6):558-68.

41. Gono T, Kawaguchi Y, Kaneko H, Nishimura K, Hanaoka M, Kataoka S, et al. Anti-NR2A antibody as a predictor for neuropsychiatric systemic lupus erythematosus. Rheumatology (Oxford, England). 2011;50(9):1578-85.

42. Fragoso-Loyo H, Cabiedes J, Orozco-Narvaez A, Davila-Maldonado L, Atisha-Fregoso Y, Diamond B, et al. Serum and cerebrospinal fluid autoantibodies in patients with neuropsychiatric lupus erythematosus. Implications for diagnosis and pathogenesis. PLoS One. 2008;3(10):e3347. 43. Tsuchiya $H$, Haga S, Takahashi $Y$, Kano $T$, Ishizaka $Y$, Mimori A. Identification of novel autoantibodies to $G A B A(B)$ receptors in patients with neuropsychiatric systemic lupus erythematosus. Rheumatology (Oxford, England). 2014;53(7):1219-28.

44. Conti F, Alessandri C, Bompane D, Bombardieri M, Spinelli FR, Rusconi AC, et al. Autoantibody profile in systemic lupus erythematosus with psychiatric manifestations: a role for anti-endothelial-cell antibodies. Arthritis research \& therapy. 2004;6(4):R366-72.

45. Croca S, Bassett P, Pericleous C, Alber KF, Latchman D, Isenberg D, et al. Serum nitrated nucleosome levels in patients with systemic lupus erythematosus: a retrospective longitudinal cohort study. Arthritis research \& therapy. 2014;16(1):R48. 
46. Gatla N, Annapureddy N, Sequeira W, Jolly M. Posterior reversible encephalopathy syndrome in systemic lupus erythematosus. Journal of clinical rheumatology : practical reports on rheumatic \& musculoskeletal diseases. 2013;19(6):334-40.

47. Kozora E, Arciniegas DB, Duggan E, West S, Brown MS, Filley CM. White matter abnormalities and working memory impairment in systemic lupus erythematosus. Cognitive and behavioral neurology : official journal of the Society for Behavioral and Cognitive Neurology. 2013;26(2):63-72.

48. Shapira-Lichter I, Vakil E, Litinsky I, Oren N, Glikmann-Johnston Y, Caspi D, et al. Learning and memory-related brain activity dynamics are altered in systemic lupus erythematosus: a functional magnetic resonance imaging study. Lupus. 2013;22(6):562-73.

49. Mak A, Ren T, Fu EH, Cheak AA, Ho RC. A prospective functional MRI study for executive function in patients with systemic lupus erythematosus without neuropsychiatric symptoms. Seminars in arthritis and rheumatism. 2012;41(6):849-58.

50. Mackay M, Bussa MP, Aranow C, Ulug AM, Volpe BT, Huerta PT, et al. Differences in regional brain activation patterns assessed by functional magnetic resonance imaging in patients with systemic lupus erythematosus stratified by disease duration. Molecular medicine (Cambridge, Mass). 2011;17(11-12):1349-56.

51. Castellino G, Govoni M, Giacuzzo S, Trotta F. Optimizing clinical monitoring of central nervous system involvement in SLE. Autoimmunity reviews. 2008;7(4):297-304.

52. Perricone C, Pendolino M, Olivieri M, Conti F, Valesini G, Alessandri C. Neuropsychiatric manifestations associated with anti-endothelial cell antibodies in systemic lupus erythematosus. The Israel Medical Association journal : IMAJ. 2015;17(3):171-8.

53. Joseph FG, Scolding NJ. Neurolupus. Practical neurology. 2010;10(1):4-15. 